\title{
Cardiovascular Effects of COVID-19 - What Do We Know and Where Should We Go?
}

\author{
Matthew Ryan, Ozan Demir, Kevin O'Gallagher and Divaka Perera \\ School of Cardiovascular Medicine and Sciences, British Heart Foundation Centre of Excellence and National Institute for Health Research \\ (NIHR) Biomedical Research Centre, King's College London, London, UK
}

A mongst the many challenges of the global pandemic, understanding the effects of coronavirus disease 2019 (COVID-19) on the cardiovascular system has emerged as a key priority. Emerging data indicate possible roles for cardiac biomarkers and cardiac imaging in the prognostic assessment of these patients, as well as implications of the vascular endothelium in the pathogenesis of the condition. From a therapeutic perspective, early data suggest that the provision of well-established treatments for cardiovascular disease, whether with angiotensin-converting enzyme inhibitors or primary angioplasty, is likely to be beneficial. These early data are of limited quality however, and robust studies are needed to address many of the key remaining questions.

\section{Keywords}

Coronavirus disease 2019, COVID-19, SARS-CoV2, myocarditis, cardiovascular disease

Disclosures: Matthew Ryan, Ozan Demir, Kevin O'Gallagher and Divaka Perera have no financial or non-financial relationships or activities to declare in relation to this article. Review Process: Double-blind peer review. Compliance with Ethics: This article is an opinion piece and does not report on new clinical data, or any studies with human or animal subjects performed by any of the authors.

Authorship: The named authors meet the International Committee of Medical Journal Editors (ICMJE) criteria for authorship of this manuscript, take responsibility for the integrity of the work as a whole, and have given final approval for the version to be published. Access: This article is freely accessible at touchCARDIO.com (c) Touch Medical Media 2020 Received: 5 May 2020

Accepted: 18 May 2020

Published Online: 18 August 2020

Citation: Heart International. 2020;14(1):16-9

Corresponding Author: Divaka Perera,

The Rayne Institute, 4th Floor Lambeth Wing,

St Thomas' Hospital, Westminster Bridge Road,

London, UK, SE1 7EH. E: divaka.perera@kcl.ac.uk

Support: No funding was received in

the publication of this article.
The current pandemic of coronavirus disease 2019 (COVID-19) is an unprecedented public health emergency, with worldwide health, social and economic implications. In contrast to other modern epidemics, little prior knowledge of the pathogen existed. Whilst this initially hampered both patient management and the public health response, we have observed a remarkable worldwide attempt to provide information to guide both clinicians and governments, with 11,478 individual publications registered in the World Health Organisation database of COVID-19 research, as of May 2020.

Early data on COVID-19 suggested poor outcomes amongst those with established cardiovascular disease and risk factors. Case reports have described patients presenting with myopericarditis and apparent acute coronary syndromes, and angiotensin-converting enzyme 2 (ACE-2) was identified as the virus's portal of entry into host cells; these observations highlight the need for research into the cardiovascular aspects of the disease. ${ }^{2}$ Whilst a substantial proportion of the published material, in this field to date, consists of opinion pieces and narrative reviews, a number of key findings have emerged and have been summarised below.

Implications of pre-existing cardiovascular disease in COVID-19

By current estimation, the case fatality rate for COVID-19 is $1.5-3.0 \%$, and the infection fatality rate is around $0.7 \%$, although this is not evenly distributed throughout the population. ${ }^{3}$ Drs Wu and McGoogan, from the Chinese Centre for Disease Control and Prevention in Beijing, reported markedly higher fatality rates in patients with cardiovascular disease (10.5\%), diabetes (7.3\%) and hypertension (6.0\%) in 72,314 cases from China. ${ }^{4}$ These findings were replicated in the Italian population. ${ }^{5}$ Closer inspection, however, revealed the prevalence of these risk factors is largely in keeping with the population prevalence of these comorbidities when analysed by age. This was also apparent in the loss of an independent effect on mortality in multivariate analyses. ${ }^{4}$

\section{Myocardial injury and COVID-19}

Zhou and colleagues first described the clinical course and predictors of outcome in 195 consecutive patients who received inpatient care for COVID-19 in Wuhan, China. ${ }^{6}$ One hundred and thirty-seven survived and 54 died, with $26 \%$ requiring intensive care unit (ICU) admission. Complete data were available for 171 patients and were used to build a regression model predicting outcomes. There are several interesting cardiovascular aspects.

Acute cardiac injury (high-sensitivity cardiac troponin I [hs-cTnl] >99th centile, $28 \mathrm{pg} / \mathrm{mL}$ in this series) was common in patients who did not survive (59\% of non-survivors versus $1 \%$ of survivors). In those who died, there was a steady upwards trend in troponin from the day of admission, though the confidence intervals (CIS) were extremely wide (Figure 1). However, it should be noted that the non-survivors were largely the critically ill ICU population, whilst the survivors were mainly cared for outside of critical care. cTnl levels were effective predictors of mortality on univariate analysis, but on multivariate analysis, the prognostic value of cTnl was lost; only age, Sequential Organ Failure Assessment (SOFA) score, and d-dimer on admission remained predictive of mortality. 
Figure 1: High-sensitivity cardiac troponin in survivors (blue) and non-survivors (red) of COVID-19 infection

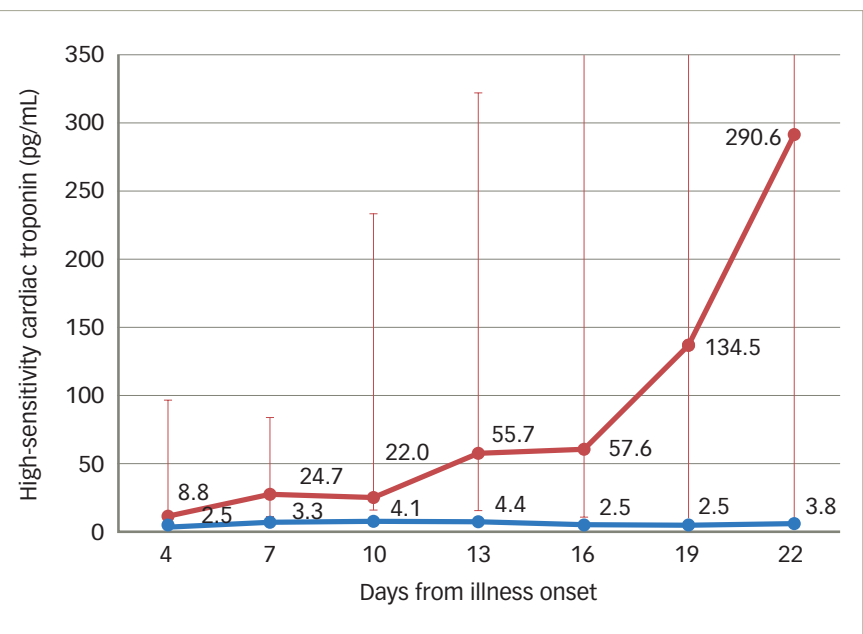

Reproduced with permission from Zhou F, et al. ${ }^{66}$

A meta-analysis of 2,389 patients from 13 studies was also published in the American Journal of Emergency Medicine. ${ }^{7}$ A hs-cTnl >99th percentile was considered to represent cardiac injury. The primary outcome was mortality and secondary outcomes were acute respiratory distress syndrome (ARDS), the need for ICU care and severe COVID-19 (any respiratory distress, saturated oxygen $\leq 93 \%$, partial pressure of arterial oxygen/fractional inspired oxygen $\left[\mathrm{PaO}_{2} / \mathrm{FiO}_{2}\right] \leq 300 \mathrm{mmHg}$, or a critical complication). High-sensitivity-cTnl was higher in patients with primary and secondary outcomes (mean difference 10.38 pg/mL [95\% Cl 4.44-16.32]). Limitations include a high degree of heterogeneity in the studies included, evidence of publication bias, and that it is difficult to know whether there was overlap in patient cohorts between studies.

Finally, a retrospective case-control study compared two independent cohorts of 73 patients with COVID pneumonitis admitted over a 7-week period in Wuhan, and 75 patients with influenza A (H1N1) ARDS admitted over a 30-month period in Beijing. ${ }^{8}$ The H1N1 group were sicker (SOFA score 5 [interquartile range 4-8] versus 2 [2-4] $p<0.001 ; \mathrm{PaO}_{2} / \mathrm{FiO}_{2}$ $<100 \mathrm{mmHg}$ in $48 \%$ versus $7 \%, \mathrm{p}<0.001$ ) and were less likely to have a history of cardiovascular disease than the group with COVID $(11 \%$ versus $32 \%$, respectively, $\mathrm{p}=0.002)$. Peak troponin I levels were higher in the H1N1 group (140 ng/L [interquartile range [20-370] versus $30 \mathrm{ng} / \mathrm{L}$ [30-50], respectively, $p=0.014$ ) but this was no longer significant when adjusted for other factors.

\section{Interpretation}

Whilst the groups in the aforementioned studies are different and this limits the certainty with which we can draw conclusions, it is likely that, whilst cardiac troponin is a prognostic marker in COVID-19, there is no evidence it provides incremental prognostic information in relation to other markers of disease severity. Furthermore, whilst there have been descriptions of individual patients presenting with overt cardiovascular pathology, the majority of even critically ill patients with COVID-19 have modest degrees of myocardial injury reflecting their overall illness severity, rather than specific cardiac involvement contributing to mortality.

\section{Imaging in COVID-19}

The role of echocardiography in COVID-19 has been uncertain, balancing the importance of assessing critically ill patients (in whom the incidence of cardiac complications is unclear) against unnecessary exposure of team members. However, a study of 120 patients from China shows the echocardiographic assessment of right ventricular (RV) function, and strain in particular, to be useful in this population. ${ }^{9}$ Several parameters of RV function - RV Iongitudinal strain (RVLS), RV fractional area change and triscuspid annular plane systolic excursion (TAPSE) - alongside male gender and the presence of ARDS were found to predict mortality, which occurred in 18 patients (15\%). RVLS and TAPSE retained their prognostic value in multivariate modelling, with RVLS providing the optimum prognostic value. Whether the observed RV dysfunction represents direct myocardial dysfunction, pulmonary thromboembolic disease or the severity of ARDS, remains to be seen.

\section{Angiotensin-converting enzyme inhibitors and angiotensin receptor blockers in COVID-19}

A recent review published in the New England Journal of Medicine elegantly summarised the background to this issue..$^{10}$ Concerns were initially raised based on select preclinical studies, stating that ACE inhibitors and angiotensin receptor blockers (ARBS) may increase ACE2 expression and provide a more ready portal of entry for COVID-19 in patients being treated with these drugs. However, little reliable data existed and none specific to COVID-19, whilst abrupt withdrawal of these drugs in high-risk patients is likely to be harmful. A clear consensus emerged across international cardiac and hypertension societies that these medications should be continued.

Subsequently, Zhang and colleagues published a retrospective nine-centre study of 1,128 patients with hypertension diagnosed with COVID-19 (188 patients on ACE inhibitors/ARBs) in Hubei Province, China from 31 December 2019 to 20 February 2020.11 Unadjusted mortality was lower in the group on ACE inhibitors/ARBs compared with those who were not (3.7\% versus $9.8 \%, p=0.01)$. There were baseline differences between the two groups (more coronary artery disease, diabetes and renal disease in the ACE inhibitor/ARB group and more stroke in the other). The authors carried out a variety of techniques to account for these differences (including an adjusted mixed-effect Cox model and propensity score-matched analyses) and concluded that the mortality benefit associated with prior ACE inhibitors/ARBs remains.

\section{Interpretation}

Notwithstanding limitations, it can probably be concluded that ACE inhibitor/ARB use is not associated with worse outcomes. The European Society of Cardiology and other international guidelines recommend continuing ACE inhibitors and ARBS in patients with COVID-19 and also in our general hypertensive and heart failure populations, for now. Randomised trials investigating the use of losartan in COVID-19 are ongoing (see ClinicalTrials.gov).

\section{Endothelial cell infection in COVID-19}

A research letter, published in The Lancet, described histological findings in three patients with COVID-19: a renal transplant recipient with coronary artery disease and hypertension who died of multiorgan failure; a female with diabetes and hypertension who died of multiorgan failure including ST-elevation myocardial infarction (STEMI), RV failure and bowel ischaemia; and a man with hypertension who had small bowel resection for ischaemia and survived.12 They found histological evidence of endothelial inflammation in the lungs, heart and submucosal vessels of the small bowel. Electron microscopy demonstrated viral inclusion in the endothelial cells of a transplanted kidney of one patient (Figure $2 A$ and $B$ ). 


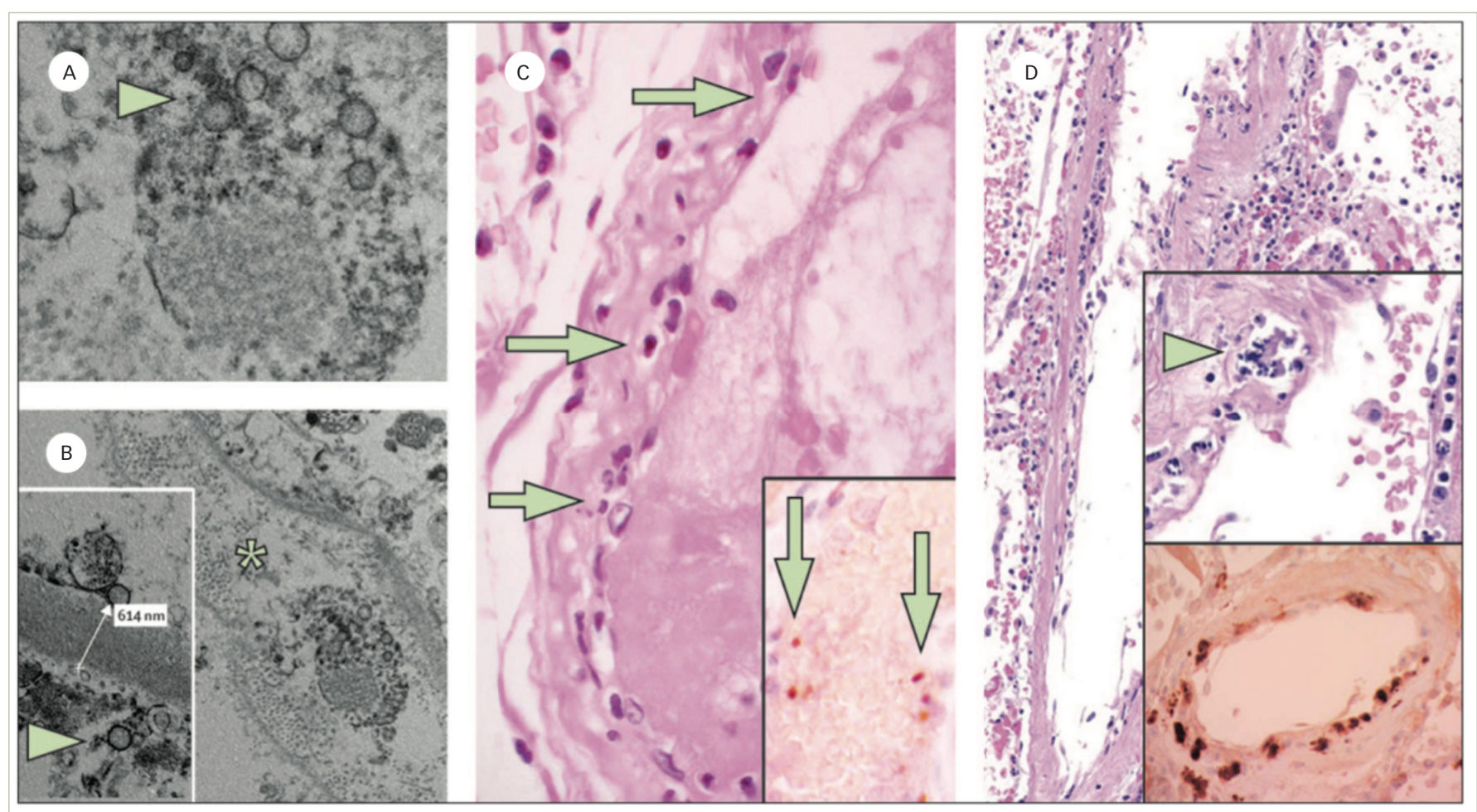

Reproduced with permission from varga $Z$, et al. ${ }^{12}$

A. B. Electron microscopy of kidney tissue shows viral inclusion bodies in a peritubular space and viral particles in endothelial cells of the glomerular capillary loops. Arrow shows aggregates of viral particles with dense circular surface and lucid centre. B. Asterisk marks peritubular space consistent with capillary containing viral particles. Inset shows the glomerular basement membrane with endothelial cell and a viral particle (arrow; about $150 \mathrm{~nm}$ diameter). C. Small bowel resection specimen of patient, stained with haematoxylin and eosin. Arrows point to dominant mononuclear cell infiltrates within the intima along the lumen of many vessels. Inset shows an immunohistochemical staining of caspase 3 in small bowel specimens from serial section of tissue described in panel D. Staining patterns were consistent with apoptosis of endothelial cells and mononuclear cells observed in the haematoxylin-eosin-stained sections, indicating that apoptosis is induced in a substantial proportion of these cells. D. Post-mortem lung specimen stained with haematoxylin and eosin showed thickened lung septa, including a large arterial vessel with mononuclear and neutrophilic infiltration (arrow in upper inset). Lower inset shows an immunohistochemical staining of caspase 3 on the same lung specimen; these staining patterns were consistent with apoptosis of endothelial cells and mononuclear cells observed in the haematoxylin-eosin-stained sections.

\section{Interpretation}

It is unclear whether endotheliitis is a direct consequence of endothelial cell infection, which may explain the relation with factors associated with endothelial dysfunction. Future post-mortem series would be instructive.

\section{Acute coronary syndromes in COVID-19}

There has been significant concern regarding patients with COVID-19 and acute coronary syndrome-like presentations, some of which have appeared to be true acute myocardial infarctions and others a number of 'STEMI mimics'. A case series of 18 patients with COVID-19 presenting as possible STEMI to New York hospitals, was published in the New England Journal of Medicine..$^{13}$ Ten out of 18 patients were labelled as having 'noncoronary myocardial injury', of whom, $90 \%$ died, compared with $50 \%$ of those with true acute myocardial infarction. Some of the most interesting data are in the supplementary appendix, including all 18 electrocardiograms (ECGS), which prove interesting to review. Half the whole cohort had normal left ventricular ejection fraction on echocardiogram. There were substantial limitations, however - only half of all patients had angiography, of whom six had percutaneous coronary intervention (PCI).

Another major concern has been a reduction in the number of patients presenting with symptoms of acute coronary syndromes. A study analysing data from nine 'high-volume' centres across several states in the USA (defined as performing $\geq 100$ primary PCI procedures per year) demonstrated a 38\% reduction in such presentations in March 2020. ${ }^{14}$ This is consistent with the $40 \%$ reduction previously reported from Spain. Speculated reasons for falling numbers of patients with STEMI included avoidance of medical care due to concerns of contracting COVID-19, misdiagnosis as suspected COVID-19, or a shift to conservative care. ${ }^{14}$

\section{Interpretation}

Whilst diagnostically challenging, the outcomes of patients with COVID-19 and suspected acute myocardial infarction are very poor, including in those with a final diagnosis of STEMI (likely because of delayed/no PCl). It is important to make a full and quick assessment (ECG, echocardiogram) of these patients and not delay angiography if felt appropriate. Simultaneously, there may be a substantial number of patients without COVID who have acute coronary syndromes who may not present acutely; whether this results in an increase in presentations with cardiogenic shock or acute heart failure remains to be seen.

\section{Review and guidance}

On 21 April 2020, the European Society of Cardiology published a document on understanding and managing cardiovascular complications of COVID-19, from pathophysiological insights to personal protective equipment. This is a pragmatic document and a good resource..$^{15}$ 


\section{Conclusions}

The pandemic has been remarkable for the rapid dissemination of data on cardiovascular involvement. Though the presence of, and risk factors for, cardiovascular disease are associated with adverse outcomes in COVID-19, the current evidence points towards these being surrogate markers for initial poor health and acute disease severity, rather than direct cardiac involvement in the majority of cases. Drugs affecting the renin-angiotensin-aldosterone system appear safe and possibly protective (though more data is needed), and the role of the vascular endothelium in the pathophysiology of COVID-19 merits investigation. The small proportion of patients who do have evidence of direct cardiac involvement merit early assessment and, if appropriate, intervention by specialists in cardiovascular medicine.
1. World Health Organisation. Global research on coronavirus disease (COVID-19). 2020. Available at: www.who.int/ emergencies/diseases/novel-coronavirus-2019/globalresearch-on-novel-coronavirus-2019-ncov (accessed 20 May 2020)

2. Hua A, O'Gallagher K, Sado D, Byrne J. Life-threatening cardiac tamponade complicating myo-pericarditis in COVID-19. Eur Heart J. 2020;41:2130.

3. Verity R, Okell LC, Dorigatti L, et al. Estimates of the severity of coronavirus disease 2019: a model-based analysis. Lancet. 2020;20:669-77.

4. WU Z, MCGoogan JM. Characteristics of and important lessons from the coronavirus disease 2019 (COVID-19) outbreak from the coronavirus disease 2019 (COVD-19) outbreak in China: summary of a report of 72314 cases from the
Chinese Center for Disease Control and Prevention. JAMA. 2020:323:1239-42.

5. Kreutz R, Algharably EAE-H, Azizi M, et al. Hypertension, the renin-angiotensin system, and the risk of lower respiratory tract infections and lung injury: implications for COVID-19.
Cardiovasc Res. 2020;116:1688-99.

6. Zhou F, Yu T, Du R, et al. Clinical course and risk factors for mortality of adult inpatients with COVID-19 in Wuhan, China: retrospective cohort study. Lancet. 2020;395:1054-62.

7. Santoso A, Pranata R, Wibowo A, et al. Cardiac injury is associated with mortality and critically ill pneumonia in COVID-19: a meta-analysis. Am J Emerg Med. 2020; doi:10.1016/j.ajem.2020.04.052 [Online ahead of print].

8. Tang X, Du R, Wang R, et al. Comparison of hospitalized patients with ARDS caused by COVID-19 and H1N1. Chest. 2020;158:195-205.

9. Li Y, Li H, Zhu S, et al. Prognostic value of right ventricular longitudinal strain in patients with COVID-19. JACC Cardiovas Imaging. 2020;doi:10.1016/j.jcmg.2020.04.014 [Online ahead

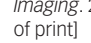

10. Vaduganathan $\mathrm{M}$, Vardeny $\mathrm{O}$, Michel $\mathrm{T}$, et al.

Renin-angiotensin-aldosterone system inhibitors in patient with COVID-19. N Eng/ J Med. 2020;382:1653-9.

11. Zhang P, Zhu L, Cai J, et al. Association of inpatient use of angiotensin converting enzyme inhibitors and angiotensin receptor blockers with mortality among patients with hypertension hospitalized with COVID-19. Circ Res 2020;126:1671-81

12. Varga Z, Flammer AJ, Steiger P, et al. Endothelial cell infection and endotheliitis in COVID-19. Lancet. 2020;395:1417-8.

13. Bangalore S, Sharma A, Slotwiner A, et al. ST-segment elevation in patients with COVID-19 - a case series. $N$ Eng/ J Med. 2020;382:2478-80.

14. Garcia S, Albaghdadi MS, Meraj PM, et al. Reduction in ST-segment elevation cardiac catherization laboratory activations in the United States during COVID-19 pandemic. I Am Coll Cardiol. 2020;75:2871-2

15. European Society of Cardiology. ESC guidelines for the diagnosis and management of CV disease during the COVID-19 pandemic. 2020. Available at: www.escardio.org/Education/ COVID-19-and-Cardiology/ESC-COVID-19-Guidance (accessed 20 May 2020). 